\title{
INVESTIGAÇÃO EPIDEMIOLÓGICA DO TÉTANO NO RIO GRANDE DO SUL, BRASIL
}

\author{
Airton Fischmann * \\ Cláudio M. da Silveira \\ Clóvis H. F. Tigre \\ José T. Fiusa Lima \\ Lucio B. Barcelos \\ Marlo Libel \\ Roberto A. Becker
}

RSPU-B $/ 307$

Fischmann, A. et al. - Investigação epidemiológica do tétano no Rio Grande do Sul, Brasil. Rev. Saúde públ., S. Paulo, 10:135-42, 1976.

RESumo: Descreve-se o comportamento epidemiológico do tétano no Rio Grande do Sul, através da análise de 136 fichas epidemiológicas de pacientes acometidos pela doença em todo o Estado. As gestantes e os escolares foram detectados como os principais grupos populacionais a serem vacinados, reduzindo-se com esta medida, a longo prazo, $80 \%$ do problema.

Unitermos: Tétano (Rio Grande do Sul). Investigação epidemiológica. Epidemiologia do tétano.

\section{N T RODU ÇÃ O}

O tétano continua sendo um grave problema de saúde pública no Rio Grande do Sul, particularmente em algumas áreas, fato demonstrável mesmo pela análise dos atestados de óbitos recolhidos pela Secretaria da Saúde.

Com a finalidade de apurar a qualidade da informação a respeito do problema, foi implantada gradativamente, a partir de 1974, uma ficha epidemiológica de investigação de casos de tétano em todo o Estado. Inicialmente a ficha foi implantada nas regiões sanitárias que contam com auxiliares de epidemiologia - Caxias do Sul, Passo Fundo, Erexim, Santa Rosa, Santa Maria, Pelotas, Bagé e Santa Cruz do Sul; a partir de junho de 1974, foi implantada nas demais regiões sanitárias - Palmeira das Missões, Cruz Alta, Santo Ângelo, Alegrete, Cachoeira do Sul, Novo Hamburgo e Porto Alegre.

O objetivo do trabalho é identificar quais os grupos mais expostos ao risco de adoecer, através da determinação do comportamento epidemiológico do tétano.

\section{MATERIAL E METODOS}

O material utilizado refere-se a 136 fichas de investigação epidemiológica utilizadas pela Secretaria da Saúde do

* Equipe técnica da Unidade de Vigilância Epidemiológica - Secretaria da Saúde do Rio Grande do Sul - Rio Grande do Sul, Brasil. 
FISCHMANN, A. et al. - Investigação epidemiológica do tétano no Rio Grande do Sul, Brasil. Rev. Saúde públ., S. Paulo, 10:135-42, 1976.

Rio Grande do Sul como instrumento do Sistema de Vigilância Epidemiológica nesse Estado.

A informação de tétano procede de nível local e chega ao nível regional através das fichas epidemiológicas preenchidas pelo técnico da Unidade Sanitária e através do certificado de óbito que é recolhido pelas Unidades Sanitárias nos cartórios.

O auxiliar de epidemiologia revisa o preenchimento das fichas, completando-as quando necessário ou mesmo preenchendo aquelas correspondentes aos óbitos, cujos certificados não vieram acompanhados deste elemento de informação.

\section{R ESULTADOS}

1. Casos de tétano por grupo etário $e$ sexo
O grupo etário mais exposto ao risco da doença é o de menores de 28 dias, com uma taxa de 193,7 casos por 100.000 . Essa taxa, significativamente elevada, aparece em geral diluída pois a taxa que se apresenta normalmente na literatura é a de casos em menores de um ano; em nossa observação este coeficiente foi de 19,3 casos por 100.000 menores de um ano (Tabela 1).

O grupo mais atingido, a seguir, é o de maiores de 60 anos, com uma taxa de 6,2 por 100,000 . Com taxas de 2,3 e 2,4 por 100.000 aparecem em terceiro lugar os grupos de 28 dias a um ano e de 5 a 15 anos incompletos, sendo o grupo etário de 30 a 45 anos incompletos o que apresenta a menor taxa (Tabela 1, Fig. 1).

Oitenta e um casos $(59,4 \%)$ eram do sexo masculino e $55(41,6 \%)$ do sexo feminino, distribuição esta similar à observada por Veronesi ${ }^{6}$ (1969). Nos grupos etários de menores de 28 dias, 5 a 15 anos e maiores de 60 anos, houve predominância de casos no sexo masculi-

T A B E L A 1

Casos de tétano por grupo etário e sexo - Rio Grande do Sul Abril de 1974 a março de $1975^{*}$

\begin{tabular}{|c|c|c|c|c|c|c|c|c|c|c|}
\hline \multirow{2}{*}{\multicolumn{2}{|c|}{ Sexo }} & \multicolumn{3}{|c|}{ Masculino } & \multicolumn{3}{|c|}{ Feminino } & \multicolumn{3}{|c|}{ Total } \\
\hline & & N.o & $\%$ & $\begin{array}{r}\text { Coef./ } \\
100.000\end{array}$ & N.o & $\%$ & $\begin{array}{r}\text { Coef./ } \\
100.000\end{array}$ & $\mathrm{~N} .0$ & $\%$ & $\begin{array}{r}\text { Coef. } \\
100.000\end{array}$ \\
\hline 0 & $1-28 d$ & 21 & 37,7 & 262,5 & 10 & 18,2 & 125,0 & 31 & 28,8 & 193,7 \\
\hline $28 d$ & 1 - $1 a$ & 1 & 1,2 & 1,1 & 3 & 5,4 & 3,3 & 4 & 2,9 & 2,4 \\
\hline 0 & - & 22 & 27,2 & 24,2 & 13 & 22,3 & 14,3 & 35 & 25,7 & 19,3 \\
\hline 1 & $1-\quad 2 a$ & 0 & 0,0 & 0,0 & 2 & 3,6 & 2,3 & 2 & 1,5 & 1,1 \\
\hline 2 & $1-5 a$ & 4 & 4,9 & 1,7 & 2 & 3,6 & 0,8 & 6 & 4,4 & 1,0 \\
\hline 5 & $1-15 a$ & 28 & 34,7 & 3,0 & 15 & 27,3 & 1,6 & 43 & 31,7 & 2,3 \\
\hline 15 & $1-30 a$ & 11 & 1,6 & 1,1 & 9 & 16,4 & 0,9 & 20 & 14,7 & 1,0 \\
\hline 30 & $1-45 a$ & 4 & 4,9 & 0,6 & 5 & 9,1 & 0,7 & 9 & 6,6 & 0,7 \\
\hline 45 & $1-60 a$ & 6 & 7,4 & 1,8 & 5 & 9,1 & 1,5 & 11 & 8,1 & 1,4 \\
\hline 60 & $e+$ & 6 & 7,4 & 7,5 & 4 & 7,3 & 5,0 & 10 & 7,3 & 6,2 \\
\hline & Total & 81 & 100,0 & 2,2 & 55 & 100,0 & 1,5 & 136 & 100,0 & 1,8 \\
\hline
\end{tabular}

* População estimada para julho de 1974.

Fonte: Unidade de Vigilância Epidemiológica -- Convênio Secretaria da Saúde-Rs/FSESP. 
FISCHMANN, A. et al. - Investigação epidemiológica do tétano no Rio Grande do Sul, Brasil. Rev. Saude puibl., S. Paulo, 10:135-42, 1976.

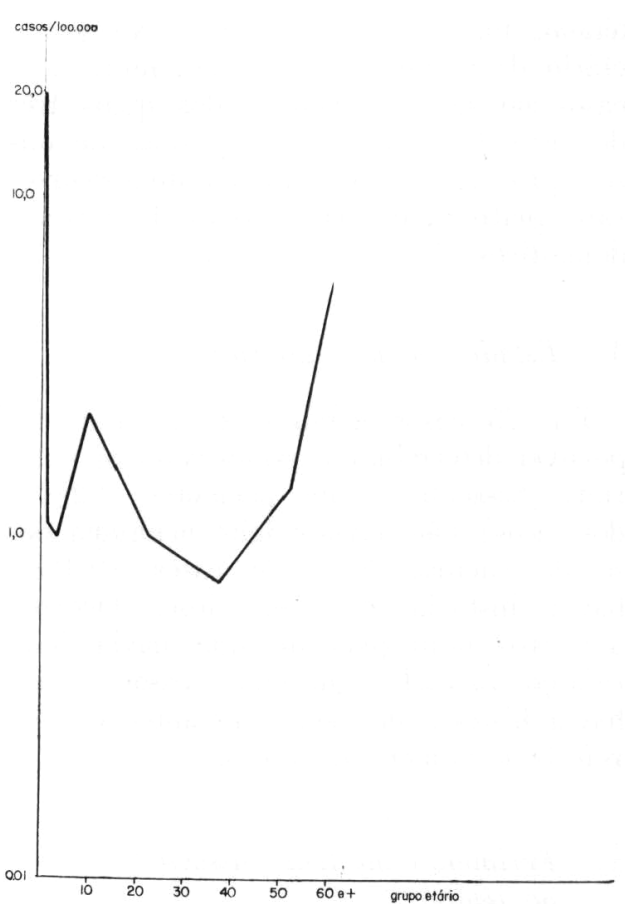

Fig. 1 - Coeficiente especifico dos casos de tétano por grupo etário. Rio Grande do Sul, abril de 1974 a março de 1975.

Fonte: Unidade de Vigilância Epidemiológica - Convênio Secretaria da Saúde-RS/ Fundação SESP.

no; nos grupos de 28 dias a um ano e um a dois anos, predomina o sexo feminino; nos demais grupos os casos por sexo se equivalem (Fig. 2).

Não existe uma explicação adequada para a nítida predominância de casos no sexo masculino em menores de 28 dias, embora este fato ocorra em várias regiōes do mundo ${ }^{1,2,4}$.

A ocorrência de maior número de casos em meninos de 5 a 15 anos, do que nas meninas deste grupo, pode ser explicado pela atividade física mais intensa desenvolvida pelas pessoas do sexo masculino nesta idade ${ }^{\mathbf{1}}$.

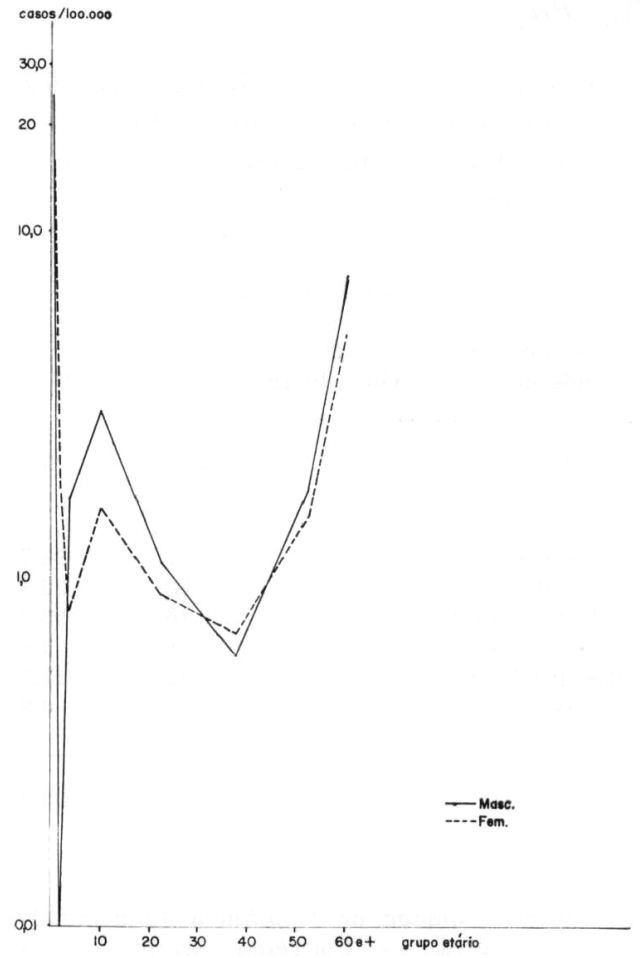

Fig. 2 - Coeficiente especifico dos casos de tétano por sexo e grupo etário. Rio Grande do Sul, abril de 1974 a março de 1975.

Fonte: Unidade de Vigilância Epidemiológica - Convênio Secretaria da Saúde-RS/ Fundação SESP.

A predominância de casos do sexo masculino nas pessoas com 60 anos ou mais, é discutida mais adiante, quando da análise das profissóes relacionadas ao tétano.

\section{Distribuição para zona rural e urbana}

Dos 136 casos, 57 ocorreram em área rural e 37 em área urbana; em 42 não se obteve informação a respeito.

A predominância dos casos em área rural se deve principalmente ao acometimento da doença em menores de 28 dias (26 casos em área rural); excluindo-se este grupo verifica-se que o tétano predomina em área urbana. 
FISCHMANN, A. et al. - Investigação epidemiológica do tétano no Rio Grande do Sul, Brasil. Rev. Saúle pübl., S. Pauio, 10:135-42, 1976.

\section{Profissão}

A Tabela 2 mostra a distribuição dos casos de tétano por grupos profissionais. (Deste estudo foram excluídos os menores de um ano).

\section{TABELA 2}

Casos de tétano segundo a profissão - Rio Grande do Sul - Abril de 1974 a marso de 1975

\begin{tabular}{lrr}
\hline \multicolumn{1}{c}{ Profissão } & Casos & $\%$ \\
\hline & & \\
\hline Estudantes & 37 & 36.7 \\
Agricultores & 23 & 22,8 \\
Afazeres domésticos & 16 & 15,9 \\
Pré-escolares * & 8 & 7,8 \\
Sem profissão & 12 & 11,9 \\
Outras & 5 & 4,9 \\
& & \\
\hline \multirow{2}{*}{ Total } & 101 & 100.0 \\
& &
\end{tabular}

De 1 a 5 anos.

Fonte: Unidade de Vigilância Epidemiológica - Convênio Secretaria da Saúde-RS/FSESP.

Com a finalidade de relacionar "profissāo" com a situação que ocasionou o ferimento, analisaram-se 44 fichas, das quais foi possivel obter esta informação. Foram excluídos os estudantes e pré-escolares.

Dos 44 casos, em $24(54,5 \%)$, a situaçāo que ocasionou o ferimento estava relacionada com o exercício da profissão. Nos casos de agricultores esta percentagem foi maior; de 21 casos em que foi possível obter informação, em $19(90,5 \%)$ o ferimento ocorreu durante o exercício da profissão. Destes dezenove casos, em dezessete $(89,5 \%)$ o ferimento ocorreu no pé. Em um caso ocorreu na mão e num outro no braço.

Das profissões listadas neste estudo, a que apresentou maior risco de lesão durante o trabalho, e conseqüentemente, de tétano, foi a de agricultor ${ }^{5}$. No grupo etário de maiores de 60 anos, houve seis casos no sexo masculino, dos quais três de agricultores e três em pessoas de outras profissões e no sexo feminino ocorreram quatro. todos em pessoas de afazeres domésticos.

\section{Estado vacinal anterior}

Em 75 casos maiores de 28 dias foi possivel determinar o estado vacinal anterior. Sessenta e um pacientes $(82,6 \%$ dos (asos) não haviam feito nenhuma dose de vacina. Em sete casos $(9,3 \%)$ havia história de uma dose. Ocorreu um caso num paciente que havia feito reforço vacinal. Em cinco casos (7\%) havia história de vacinação anterior, sem referir o número de doses.

\section{Ferimento ou lesão associada ao tétano}

Excluindo o grupo de menores de 28 dias, observou-se que em 93 casos foi possivel determinar a lesão associada ao tétano. Os tipos de ferimentos ocorridos estão expressos na Tabela 3.

T A B E L A 3

Tipos de ferimentos associados ao tétano Rio Grande do sul - Abril de 1974 a março de 1975

\begin{tabular}{lrr}
\hline \multicolumn{1}{c}{ Tipo de ferimento } & Total & $\%$ \\
\hline Punctório & & - \\
Laceração & 44 & 47,4 \\
Escoriacão & 20 & 21,6 \\
Queimadura & 10 & 10,7 \\
Contusão & 5 & 5,4 \\
Pós-cirúrgica & 4 & 4,3 \\
Corto-contuso & 4 & 4,3 \\
Projétil de arma de fogo & 2 & 2,2 \\
& 1 & 1,1 \\
\hline \multirow{2}{*}{ Total } & & \\
\end{tabular}

Fonte: Unidade de Vigilância Epidemiológica-Rs. 
FISCHMAxir, A. et al. - Investigasão epidemiológica do tétano no Rio Grande do Sul, Bra. sil Rer. Sande publ., S Paulo, 10:135-42, 1976.

Quanto à região do corpo afetada, obserrou-se que o ferimento ocorreu no pé em 56 casos 160.2 $c$ ); na coxa e na perna em 20 (21.5' c) : na cabeça e pescoco $+(4.3(c)$ : no tronco $+(4.3(r)$; na mão $+(4.3 \%)$; hraço e antehraço 2 (2.1\%) e em lugares múltiplos em 2 pacientes $(2.1 \%$ c).

\section{Periodo de incubação}

As freqüencias encontradas para o período de incuhação do tétano no grupo etário a partir de 28 dias, foram as seguintes:

$$
\begin{aligned}
& 0^{\prime} \text { - } 2 \text { dias } 4 \text { casos } \\
& 2 \text { - } 4 \text { dias } 4 \text { casos } \\
& 4 \text { - } 6 \text { dias } 12 \text { casos } \\
& 6 \text { ! } 8 \text { dias } 15 \text { casos } \\
& 8 \text { - } 10 \text { dias } 17 \text { casos } \\
& 10,-12 \text { dias } 6 \text { casos } \\
& 12 \text { - } 14 \text { dias } 4 \text { casos } \\
& 14-16 \text { dias } 7 \text { casos } \\
& 16 \text { - } 18 \text { dias } 1 \text { caso } \\
& 18 \mathrm{e}+\text { zero } \\
& \text { Indeterminado } 35 \\
& \text { Total } 105 \text { casos }
\end{aligned}
$$

Como se pode depreender da relação acima. o período de incubação que ocorreu com maior freqüência nos casos de tétano neste grupo foi o de oito a dez dias. Para o tétano em menores de 28 dias. o período de incubação mais freqüente também foi de oito a dez dias.

\section{Administração préria do soro antitetânico}

Dos 105 casos maiores de 28 dias, em $56 \quad(53.3 \%)$ havia referência de administração de soro antitetânico prévio; em $36(34.3 \%)$ não foi administrado soro e em $23(21.4 \%)$ esta informação era ignorada. Dos 56 casos em que foi administrado o soro antitetânico, em 40 $(71.4 \%)$ a administração foi feita mais de 48 horas após o ferimento: em sete (12.5'c). 25 a 18 horas: em três 6 a 24 horas; em dois menos de 6 horas após e em quatro o dado era ignorado.

\section{Quadro clínico}

Os três principais sinais ocorridos em todas as idades foram convulsóes, trismo e rigidez muscular generalizada (RIIG).

Em 54.4\% dos casos ocorreram os três sinais. Em 19.8\% ocorreram trismo e RMG. Em 8.8\% ocorreram convulsões e RMG. Em $7.3 \%$ a RIIG ocorreu isoladamente. As demais alternatiras, ocorridas em conjunto ou separadamente. representaram $9.7 \%$ do total.

\section{Hospitalização}

Todos os casos, com exceção de um, foram hospitalizados; este fato permite, em parte, uma avaliação mais precisa da letalidade, pois a assistência médica prestada a pacientes foi, presumirelmente, semelhante em todo o Estado.

\section{Letalidade}

A letalidade em todas as idades, foi de $52.2^{\circ} \mathrm{c}$. Para maiores de 28 dias foi de $48 C^{\circ}$ e para menores de 28 dias foi de $93.5 \%$.

A distribuição da letalidade em todos os grupos etários, está expressa na Tabela 4 e Figura 3.

\section{Menores de 28 dias}

A) Vacinação antitetânica da mãe

Dos 31 casos ocorridos em menores de 28 dias. em rinte ohteve-se história vacinal antitetânica da mãe. Dezoito mães $190 \%$ ) nunca receberam anatox tetânico. Das duas mães racinadas previamente. uma delas referia ter feito uma dose cinco anos antes do parto e a outra. embora referisse ter sido racinada. ignora- 
FISCHMANN, A. et al. - Investigação epidemiológica do tétano no Rio Grande do Sul, Brasil. Rev. Sauide puibl., S. Paulo, 10:135-42, 1976.

TABELA 4

Letalldade do tétano por grupo etárlo - Rio Grande do sul - Abril de 1974 a maio de 1975

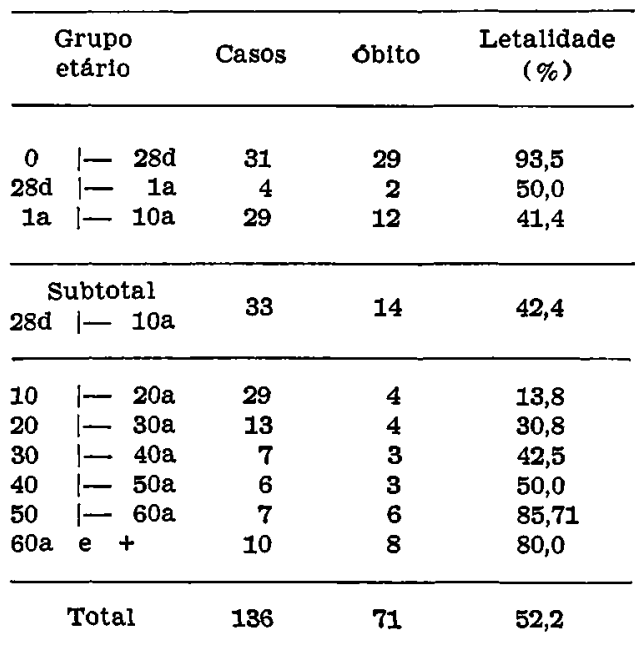

Fonte: Unidade de Vigilância Epidemiológica-Rs.

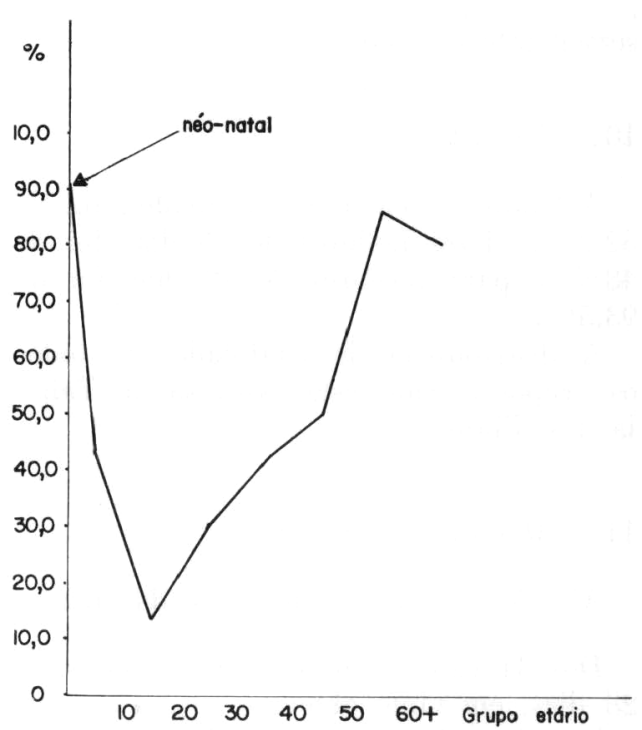

Fig. 3 - Taxa de letalidade de tétano, por grupo etário. Rio Grande do Sul, abril de 1974 a março de 1975.

Fonte: Unidade de Vigilância Epldemiológica - Convênio Secretaria da Saúde-RS/ Fundação SESP. va o número de doses recebidas, bem como a época de administração.

\section{B) Local do parto}

Vinte e nove casos de tétano neonatal originaram-se de partos domiciliares $(93,5 \%)$. Em dois casos $(6,5 \%)$ o parto foi hospitalar.

\section{C) Atendimento do parto}

Dos 31 casos de tétano neonatal, em 23 oportunidades $(74,2 \%)$ o parto foi atendido por curiosa; em três $(9,6 \%)$ por vizinha; em duas vezes $(6,4 \%)$ por auxiliar de enfermagem (os dois partos hospitalares); houve ainda dois partos atendidos por familiares e num outro a informação é ignorada.

\section{DISCUSSA O}

Através do estudo de 136 fichas epidemiológicas de tétano, foi possivel determinar vários pontos importantes quanto ao comportamento epidemiológico desta doença no Rio Grande do Sul.

Observou-se que $60,8 \%$ dos casos ocorreram no sexo masculino, com nítida predominância dos casos do sexo masculino no grupo de menores de 28 dias e de cinco a quinze anos incompletos.

O grupo de menores de 28 dias contribuiu com $22,8 \%$ do total de casos ou com uma taxa de 17,2 por 100.000 habitantes (taxa mais elevada do que nos demais grupos). Este grupo contribuiu também com $40,8 \%$ do total de óbitos. Este dado é semelhante ao determinado no estudo da mortalidade do tétano no Rio Grande do $\mathrm{Sul}^{1}$ onde os óbitos de menores de 28 dias representavam $44,8 \%$ do total.

Em $93,5 \%$ dos casos de tétano neonatal, o parto foi domiciliar e em $86,3 \%$ das vezes ocorreu em área rural. Esta 
FISCHMANN, A. et al. - Investigação epidemiológica do tétano no Rio Grande do Sul, Brasil. Rev. Saúde públ., S. Paulo, 10:135-42, 1976.

informação é importante para a determinação da estratégia no combate ao problema.

A ocupação em que ocorreu maior número de casos foi a de estudante $(34,3 \%)$, vindo em seguida os casos em agricultores $(22 \%)$, sendo que entre estes últimos, o ferimento se produziu durante o desempenho da atividade profissional em $90,5 \%$ das oportunidades; essa foi considerada, pois, a profissão de maior risco de contrair o tétano no Rio Grande do Sul.

o ferimento que ocorreu com maior frequiência foi o de tipo punctório e em segundo lugar a laceração; fato semelhante foi observado nos Estados Unidos ${ }^{2}$.

As regióes do corpo mais afetadas foram os pés $(60,2 \%)$ e coxas e pernas $(21,5 \%)$; ou seja, em $81,7 \%$ das vezes o ferimento ocorreu nos membros inferiores.

$O$ período de incubação que ocorreu mais frequientemente para todos os grupos etários, foi de oito a dez dias de duração.

Quanto ao estado vacinal anterior, observou-se que a grande maioria dos casos de tétano ocorreu em não vacinados $(82,6 \%)$.

A taxa de letalidade foi mais elevada no grupo neonatal, atingindo $93,5 \%$; decresceu progressivamente até $13,8 \%$ no grupo de 10 a 20 anos incompletos (a menor taxa). Após este grupo ela ascende paulatinamente, sendo de $85,71 \%$ no grupo de 50 a 60 anos incompletos e de $80 \%$ nos maiores de 60 anos.
CONCLUSOES

Através deste estudo foi possível esboçar o comportamento epidemiológico do tétano no Rio Grande do Sul, determinando-se vários grupos populacionais prioritários para os programas de vacinação.

Pode-se propor a vacinação de determinadas faixas da população contra o tétano, como a de gestantes e escolares sem a necessidade de se vacinar toda uma população. Sabe-se também que o grupo populacional de gestantes é um dos mais difíceis de se alcançar devido a dificuldade de localização e mobilização deste grupo, quando desejamos obter uma cobertura adequada de assistência pré-natal. Daí a necessidade de se fortalecer este tipo de programa visando atingir as populações de gestantes em áreas rurais, quer pelo atendimento direto introduzindo a vacinação no interior do município, quer pela mobilização e capacitação das curiosas, ou ainda pela obtenção de facilidades visando o atendimento do maior número possível de partos hospitalares.

Desta forma, parece perfeitamente adequado o desenvolvimento de atividades visando a cobertura de determinados grupos populacionais com vacina antitetânica; pode-se reduzir a curto prazo, em $60 \%$ a magnitude do dano e a longo prazo em aproximadamente $80 \% \mathrm{com}$ a vacinação de $32 \%$ da população: 1.890 .396 crianças de 5 a 14 anos mais 446.434 mulheres de 15 a 30 anos residentes em área rural, no Rio Grande do Sul.

RSPU-B/307

FischmanN, A. et al. - [Epidemiological Investigation of Tetanus in Rio Grande do Sul, Brazil]. Rev. Saúde públ., S, Paulo, 10:135-42, 1976.

SUMMary: The epidemiologic situation of tetanus in Rio Grande do Sul is described, based on the analysis of 136 individual case reports. It was observed that the most important groups to be vaccinated were pregnant women and school-age children. By developing this strategy an $80 \%$ reduction of the problem can be achieved over a long period.

UNITERMS: Tetanus; Rio Grande do Sul (Brazil). Epidemiological investigation. Epidemiology, tetanus. 
FISCHMANN, A. et al. - Investigaçāo epidemiológica do tétano no Rio Grande do Sul, Brasil. Rev. Saude pribl., S Paulo, 10:135-42, 1976.

\section{REFERENCIAS BIBLIOGRÁFICAS}

1. BOLETIM EPIDEMIOLÓGICO, (Ministério da Saúde. Divisão de Epidemiologia, Estatística e Informasão). Rio de Janeiro, $\gamma(2): 11,1975$.

2. CENTER FOR DISEASE CONTROL Tetanus Surveillance. Atlanta, Ga. 1974. (Report 4).

3. EDELWEISS, E. L. - Quelques suggestions pour le combat an tetanus en Rio Grande do Sul (Brésil). Amsterdam, Institut Royal de Medicine Tropicale, 1966. (Monographie de conclusion. Cours international de Promotion de la Sauté).
4. NATIONAL COMUNICABLE DISEASES - Tetanus Surveillance. Atlanta, Ga., 1970. (Report 3).

5. VERONESI, R. - Tétano. In: VERONESI, R., ed. - Doencas infecciosas $e$ parasitáras. 3.a ed. Rio de Janeiro, Guanabara Koogan, 1974. p. 481.

6. VERONESI, R. - Tétano. In: VERoNESI, R., ed. - Doenças inferciosas $e$ parasitárias. $4 .^{\mathbf{a}}$ ed. Rio de Janeiro, Guanabara Koogan, 1969. p. 499.

Recebido para publicasio em 04/09/1975 A provado para publicasio em $05 / 01 / 1976$ 